\title{
Quantitation of 11 alkylamines in atmospheric samples: separating structural isomers by ion chromatography
}

Bryan K. Place et al.

Correspondence to: Trevor C. VandenBoer (tvandenboer@mun.ca)

The copyright of individual parts of the supplement might differ from the CC-BY 3.0 licence. 


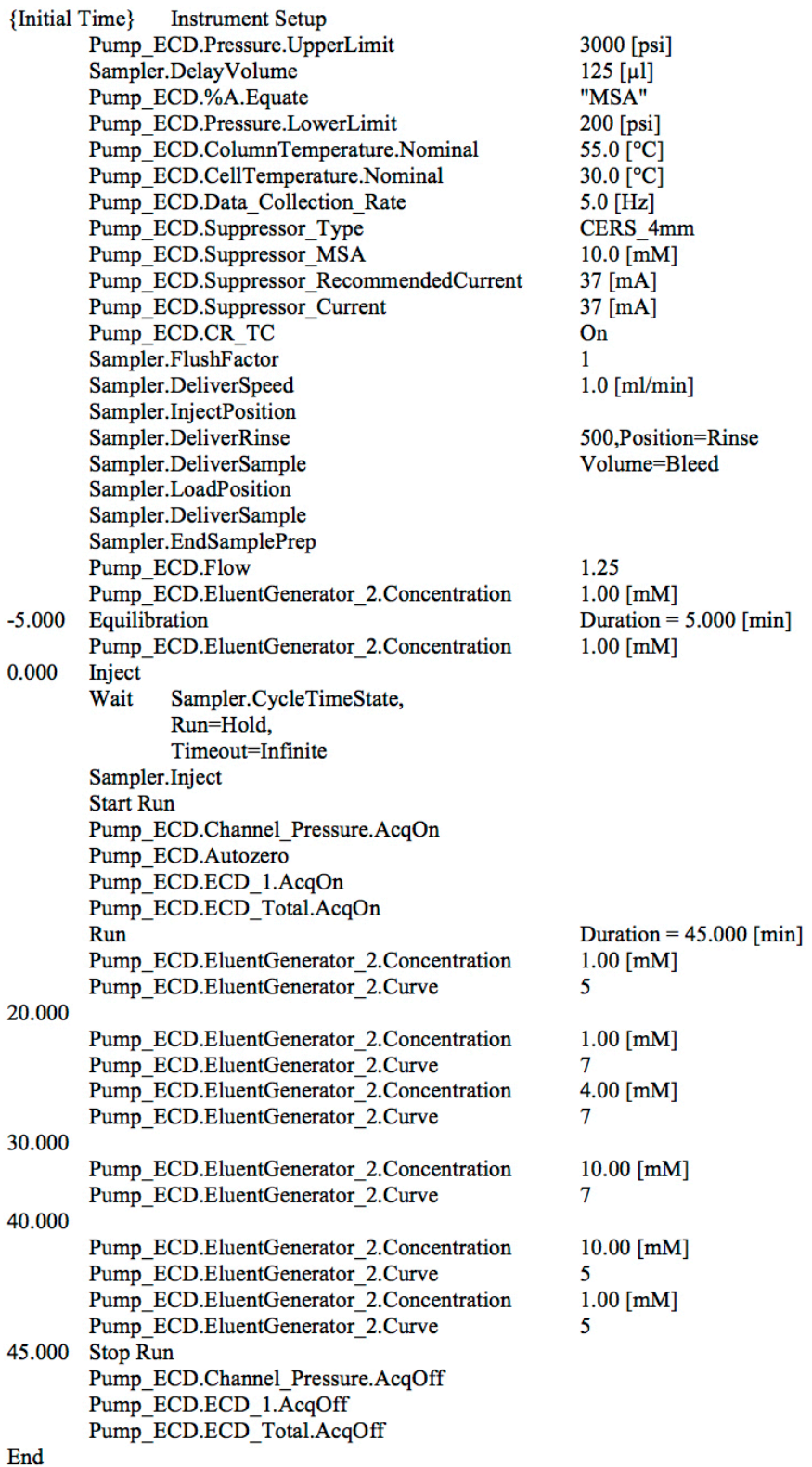

Fig S1. Chromeleon method data file for final gradient method at $55^{\circ} \mathrm{C}$ 


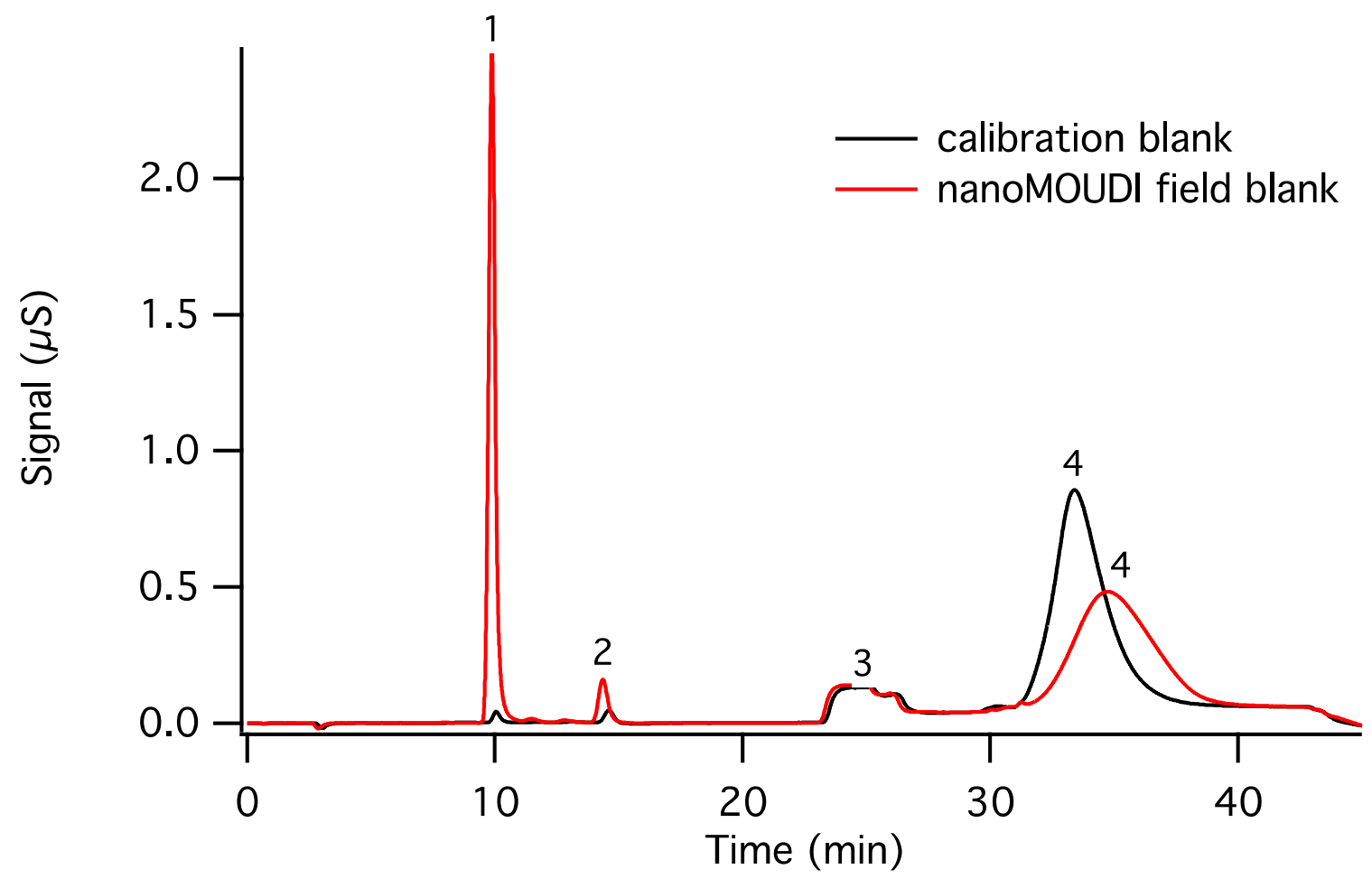

Fig S2. Sample chromatograms of a calibration blank and a size-resolved BB MOUDI foil substrate field blank. The peaks labelled above are as follows: $\mathrm{Na}^{+}(1), \mathrm{K}^{+}(2)$, System peak (3), and $\mathrm{Ca}^{2+}(4)$. 

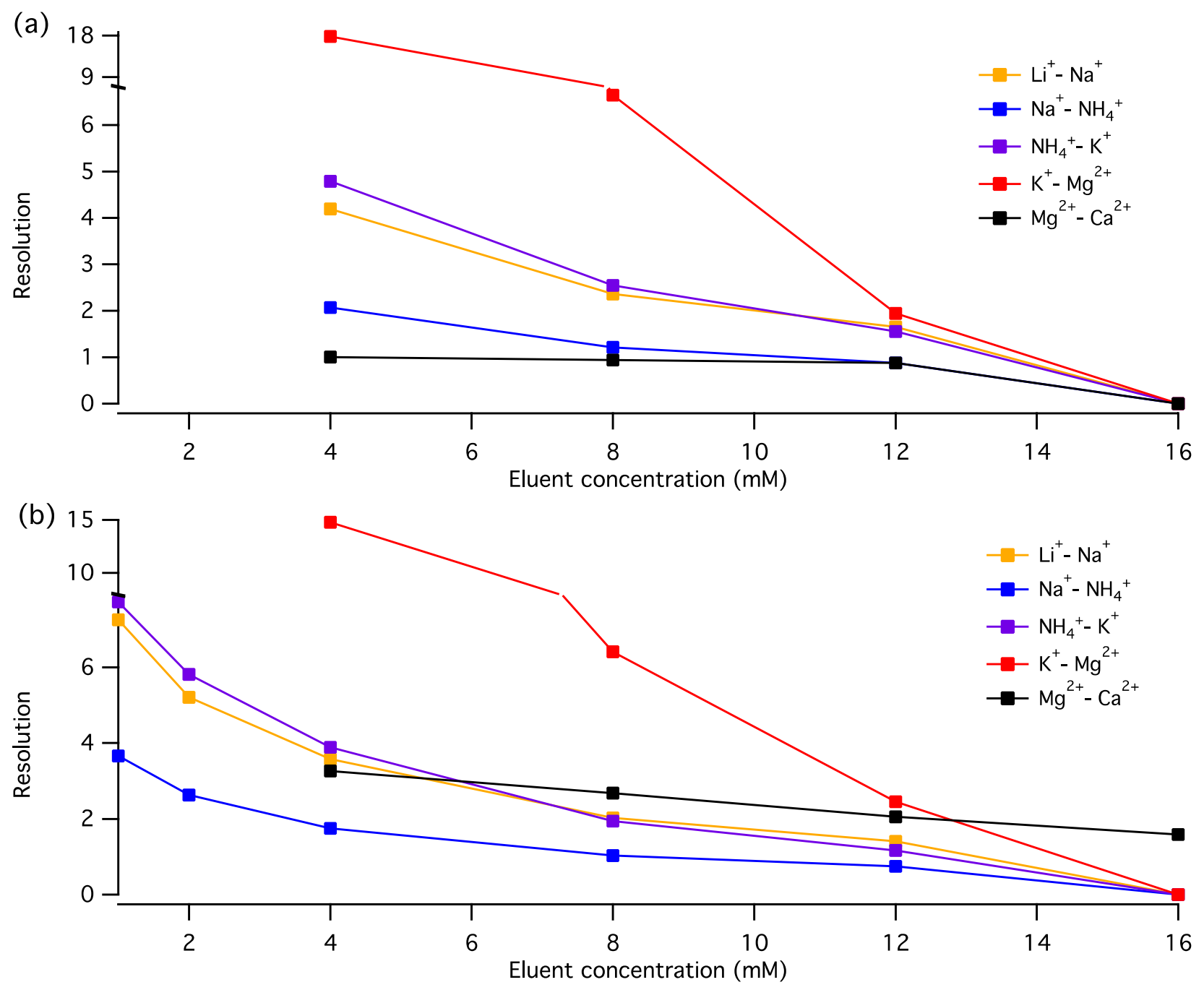

5 Fig S3. (a) Resolution of the six inorganic cation peak pairs using isocratic eluent methods at a flow rate of $0.75 \mathrm{ml} \mathrm{min}^{-1}$. (b) Resolution of the six inorganic cation peak pairs using isocratic eluent methods at a flow rate of $1.25 \mathrm{ml} \mathrm{min}^{-1}$. The resolution axis is split to indicate eluent concentrations where dramatic increases in separation occurred. 

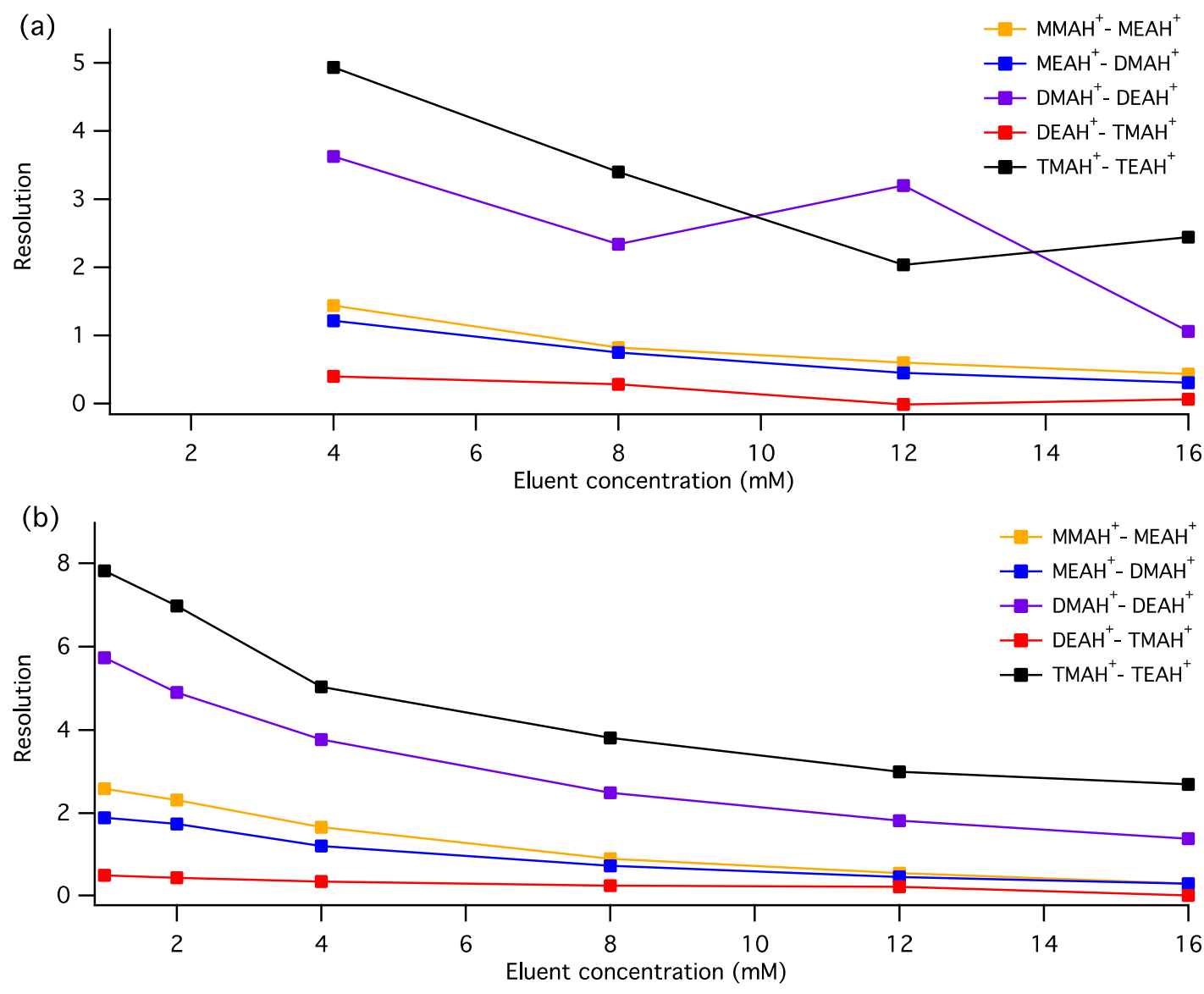

Fig S4. (a) Resolution of the six alkyl amine cation peak pairs using isocratic eluent methods at a flow rate of $0.75 \mathrm{ml} \mathrm{min}^{-1}$. 5 (b) Resolution of the six alkyl amine cation peak pairs using isocratic eluent methods at a flow rate of $1.25 \mathrm{ml} \mathrm{min}^{-1}$. 

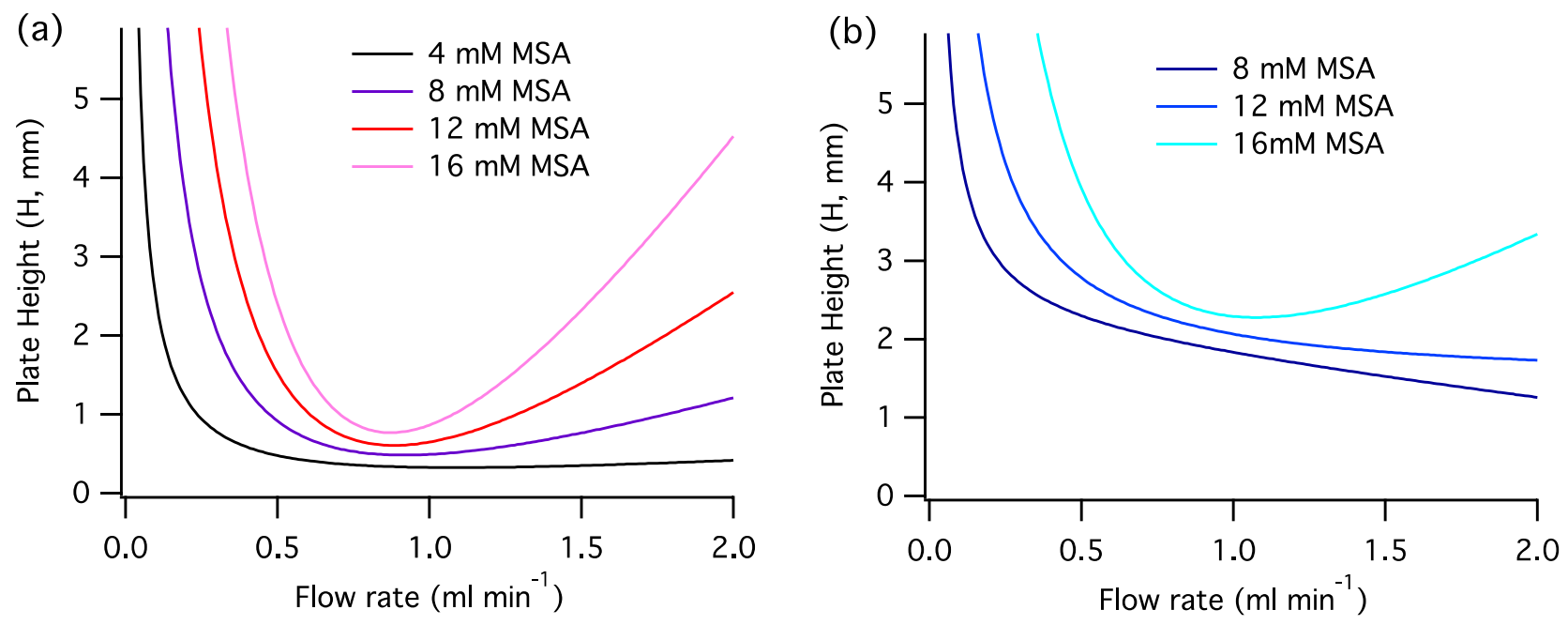

Fig S5. Calculated Van Deemter plots for the isocratic elutions of (a) $\mathrm{MMAH}^{+}$and (b) $\mathrm{TEAH}^{+}$at various MSA eluent concentrations and flow rates.

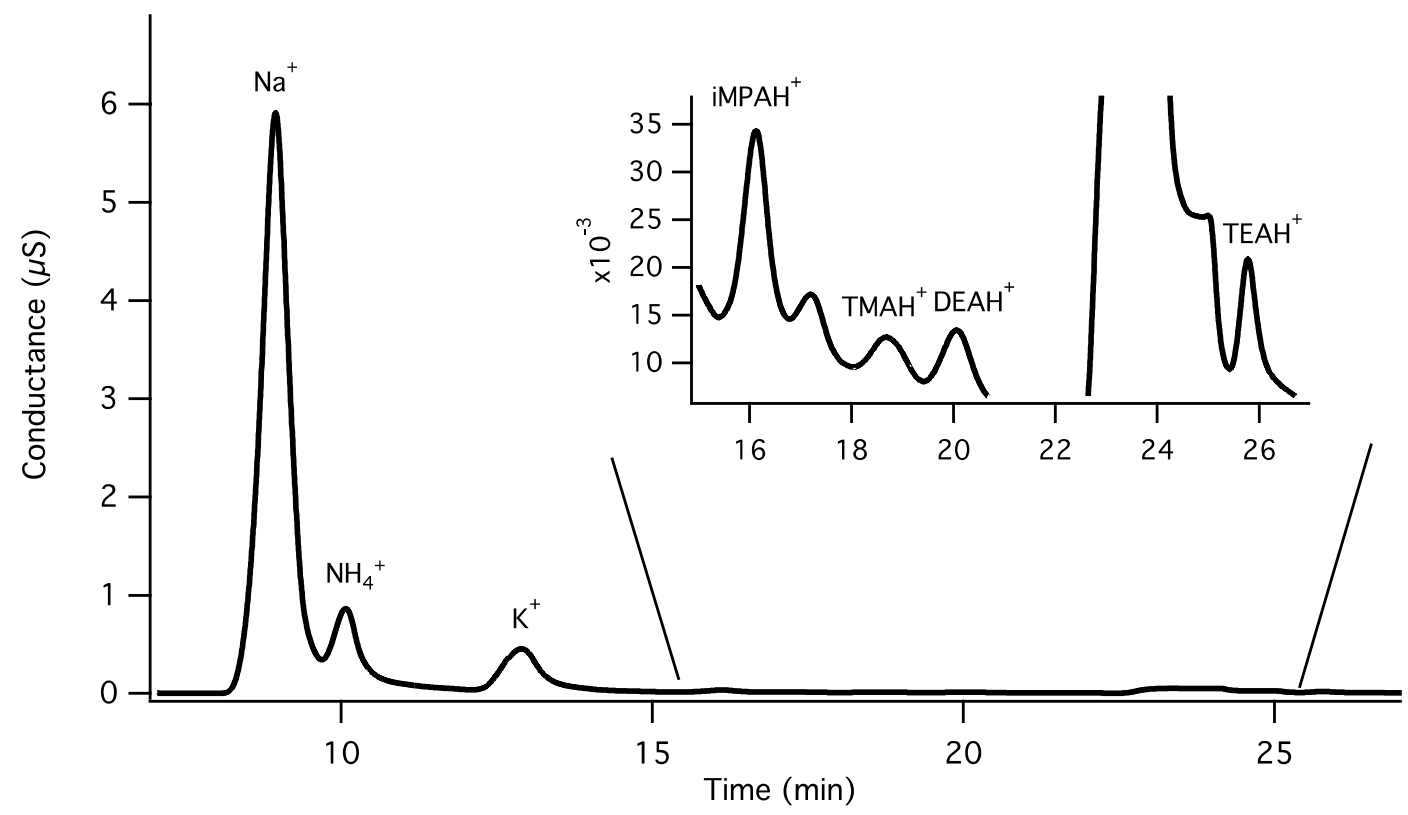

Fig S6. A chromatogram from an extracted $\mathrm{PM}_{2.5}$ sample collected during a biomass-burning event in British Columbia at the Burnaby Kensington Park site. 
Table S1. Comparison of methyl and ethyl amine external and standard addition calibration slopes and retention times ( $\mathrm{t}_{\mathrm{r}}$ )

\begin{tabular}{lccccc}
\hline Analyte & $\begin{array}{c}\text { External } \\
\left(\boldsymbol{\mu} \mathbf{S}^{*} \mathbf{m i n} \mathbf{~ m o l}^{-\mathbf{1}}\right)\end{array}$ & $\begin{array}{c}\text { Standard addition } \\
\left(\boldsymbol{\mu S} \mathbf{S m i n ~ m o l}^{-\mathbf{1}}\right)\end{array}$ & $\begin{array}{c}\text { Difference } \\
(\mathbf{\%})\end{array}$ & $\begin{array}{c}\text { External } \mathbf{t}_{\mathbf{r}} \\
\text { range }(\mathbf{m i n})\end{array}$ & $\begin{array}{c}\text { Standard addition } \mathbf{t}_{\mathbf{r}} \\
\text { range }(\mathbf{m i n})\end{array}$ \\
\hline $\mathrm{MMAH}^{+}$ & $0.41 \mathrm{E} 08$ & $0.42 \mathrm{E} 08$ & 2 & $9.0-9.4$ & $9.3-9.6$ \\
$\mathrm{DMAH}^{+}$ & $0.98 \mathrm{E} 08$ & $1.01 \mathrm{E} 08$ & 3 & $10.4-10.8$ & $10.6-11.0$ \\
$\mathrm{TMAH}^{+}$ & $0.13 \mathrm{E} 08$ & $0.13 \mathrm{E} 08$ & 0 & $13.2-13.5$ & $13.4-13.8$ \\
$\mathrm{MEAH}^{+}$ & $0.47 \mathrm{E} 08$ & $0.51 \mathrm{E} 08$ & 8 & $10.1-10.5$ & $10.3-10.8$ \\
$\mathrm{DEAH}^{+}$ & $1.06 \mathrm{E} 08$ & $1.08 \mathrm{E} 08$ & 2 & $13.9-14.2$ & $14.1-14.5$ \\
$\mathrm{TEAH}^{+}$ & $0.57 \mathrm{E} 08$ & $0.57 \mathrm{E} 08$ & 0 & $23.6-24.0$ & $23.9-24.2$ \\
\hline
\end{tabular}

Table S2. Analytical performance of other IC methods used for the determination of atmospheric methyl and ethyl amines.

\begin{tabular}{|c|c|c|c|c|c|c|}
\hline Analyte & $\begin{array}{c}\text { Detection } \\
\text { method }\end{array}$ & $\begin{array}{l}\text { Pre- } \\
\text { conc }\end{array}$ & Column & LOD (pg) & $\begin{array}{c}\text { Precision } \\
(\%)\end{array}$ & Reference \\
\hline \multirow[t]{9}{*}{$\mathrm{MMAH}^{+}$} & $\mathrm{CD}$ & Yes & CS10 & 31 & $2-2.7$ & Chang et al., 2003 \\
\hline & $\mathrm{CD}$ & Yes & CS12A & 18 & 4.5 & VandenBoer et al., 2012 \\
\hline & $\mathrm{CD}$ & No & CS14 & 2500 & 3.8 & Verriele et al., 2012 \\
\hline & MS & No & CS14 & 500 & 5.8 & Verriele et al., 2012 \\
\hline & CD & Yes & CS17 & 540 & 4.8 & VandenBoer et al., 2012 \\
\hline & CD & Yes & CS19 & $30-650$ & 5 & This work \\
\hline & CD & No & Metrosep C2 & 21500 & 0.4 & Erupe et al., 2010 \\
\hline & $\mathrm{CD}$ & Yes & Metrosep C4 & 2100 & 12.2 & Huang et al., 2014 \\
\hline & $\mathrm{CD}$ & No & Metrosep C4 & 160 & 7.3 & Dawson et al., 2014 \\
\hline \multirow[t]{10}{*}{$\mathrm{DMAH}^{+}$} & CD & Yes & CS10 & 40 & $2-2.7$ & Chang et al., 2003 \\
\hline & CD & Yes & CS12A & 25 & 1 & VandenBoer et al., 2012 \\
\hline & CD & No & CS14 & 4000 & 10.5 & Verriele et al., 2012 \\
\hline & MS & No & CS14 & 150 & 11.4 & Verriele et al., 2012 \\
\hline & $\mathrm{CD}$ & Yes & CS17 & 870 & 14 & VandenBoer et al., 2012 \\
\hline & CD & No & CS17 & 1500 & 1.2 & Li et al., 2009 \\
\hline & CD & Yes & CS19 & $40-650$ & 5 & This work \\
\hline & CD & No & Metrosep C2 & 23000 & 1.4 & Erupe et al., 2010 \\
\hline & CD & Yes & Metrosep C4 & 3800 & 15.7 & Huang et al., 2014 \\
\hline & $\mathrm{CD}$ & No & Metrosep C4 & 320 & 1.1 & Dawson et al., 2014 \\
\hline \multirow[t]{7}{*}{$\mathrm{TMAH}^{+}$} & CD & Yes & CS10 & 26 & $2-2.7$ & Chang et al., 2003 \\
\hline & $\mathrm{CD}$ & Yes & CS12A & 220 & 1 & VandenBoer et al., 2012 \\
\hline & $\mathrm{CD}$ & No & CS14 & 2500 & N/A & Verriele et al., 2012 \\
\hline & MS & No & CS14 & 500 & 12.2 & Verriele et al., 2012 \\
\hline & CD & Yes & CS17 & 1580 & 3.3 & VandenBoer et al., 2012 \\
\hline & $\mathrm{CD}$ & No & CS17 & 2000 & 3.5 & Li et al., 2009 \\
\hline & $\mathrm{CD}$ & Yes & CS19 & $300-1200$ & 16 & This work \\
\hline
\end{tabular}




\begin{tabular}{ccccccc}
\hline \multirow{5}{*}{ MEAH $^{+}$} & CD & No & Metrosep C2 & 38000 & 1.1 & Erupe et al., 2010 \\
& CD & No & Metrosep C4 & 970 & 6.1 & Dawson et al., 2014 \\
& CD & Yes & CS10 & 37 & $2-2.7$ & Chang et al., 2003 \\
& CD & Nos & CS12A & 33 & 12 & VandenBoer et al., 2012 \\
& MS & No & CS14 & 1000 & 5.1 & Verriele et al., 2012 \\
& CD & Yes & CS17 & 500 & 7.9 & Verriele et al., 2012 \\
& CD & Yes & CS19 & 790 & 10 & VandenBoer et al., 2012 \\
& CD & Yes & Metrosep C4 & 2200 & 4.3 & Huang et al., 2014 \\
DEAH $^{+}$ & CD & Yes & CS12A & 195 & 14 & VandenBoer et al., 2012 \\
& CD & No & CS14 & N/A & N/A & Verriele et al., 2012 \\
& MS & No & CS14 & 35 & 9 & Verriele et al., 2012 \\
& CD & Yes & CS17 & 1140 & 3.5 & VandenBoer et al., 2012 \\
& CD & Yes & CS19 & $100-800$ & 8 & This work \\
& CD & Yes & Metrosep C4 & 4100 & 4.6 & Huang et al., 2014 \\
TEAH $^{+}$ & CD & Yes & CS12A & 32000 & 2 & VandenBoer et al., 2012 \\
& CD & Yes & CS17 & 1870 & 5.9 & VandenBoer et al., 2012 \\
& CD & Yes & CS19 & $500-1400$ & 12 & This work \\
& CD & Yes & Metrosep C4 & 15900 & 5.1 & Huang et al., 2014 \\
\hline
\end{tabular}

Table S3. Mass loadings of amines and ammonium in size-resolved particle diameter $\left(D_{p}\right)$ samples from an aged biomass burning plume sampled in St. John’s, Newfoundland on July 6, 2013.

\begin{tabular}{cccccccc}
\hline \multirow{2}{*}{$\begin{array}{c}\boldsymbol{7} \\
(\boldsymbol{\mu m})\end{array}$} & $\mathbf{N H}_{\mathbf{4}}{ }^{+}$ & $\mathbf{M M A H}^{+}$ & $\mathbf{D M A H}^{+}$ & $\mathbf{T M A H}^{+}$ & $\mathbf{M E A H}^{+}$ & DEAH $^{+}$ & TEAH $^{+}$ \\
\hline $10-18$ & $\mathrm{BDL}$ & $2.0 \pm 0.2$ & $0.6 \pm 0.2$ & $\mathrm{BDL}$ & $\mathrm{BDL}$ & $3 \pm 1$ & $2 \pm 1$ \\
$5.6-10$ & $\mathrm{BDL}$ & $\mathrm{BDL}$ & $0.7 \pm 0.3$ & $2 \pm 2$ & $\mathrm{BDL}$ & $2.2 \pm 0.9$ & $2 \pm 1$ \\
$3.2-5.6$ & $\mathrm{BDL}$ & $0.11 \pm 0.03$ & $0.4 \pm 0.1$ & $\mathrm{BDL}$ & $\mathrm{BDL}$ & $\mathrm{BDL}$ & $\mathrm{BDL}$ \\
$1.8-3.2$ & $\mathrm{BDL}$ & $0.10 \pm 0.03$ & $0.25 \pm 0.09$ & $2 \pm 1$ & $\mathrm{BDL}$ & $1.4 \pm 0.6$ & $1.3 \pm 0.7$ \\
$1-1.8$ & $\mathrm{BDL}$ & $2.9 \pm 0.8$ & $3 \pm 1$ & $3 \pm 2$ & $\mathrm{BDL}$ & $27 \pm 4$ & $4 \pm 2$ \\
$0.56-1$ & $719 \pm 7$ & $1.4 \pm 0.4$ & $190 \pm 4$ & $5 \pm 3$ & $0.4 \pm 0.2$ & $1300 \pm 200$ & $2 \pm 1$ \\
$0.32-0.56$ & $443 \pm 4$ & $11 \pm 3$ & $208 \pm 4$ & $4 \pm 3$ & $0.21 \pm 0.08$ & $1300 \pm 200$ & $4 \pm 2$ \\
$0.18-0.32$ & $236 \pm 2$ & $6 \pm 2$ & $80 \pm 2$ & $\mathrm{BDL}$ & $\mathrm{BDL}$ & $560 \pm 90$ & $2 \pm 1$ \\
$0.10-0.18$ & $30 \pm 50$ & $0.4 \pm 0.1$ & $30 \pm 10$ & $\mathrm{BDL}$ & $0.6 \pm 0.2$ & $200 \pm 30$ & $\mathrm{BDL}$
\end{tabular}




$\begin{array}{cccccccc}0.056-0.10 & 20 \pm 30 & 3 \pm 1 & 6 \pm 2 & 4 \pm 3 & \text { BDL } & 58 \pm 9 & 3 \pm 2 \\ 0.032-0.056 & 40 \pm 70 & 0.11 \pm 0.03 & 7 \pm 3 & \text { BDL } & \text { BDL } & 49 \pm 8 & \text { BDL } \\ 0.018-0.032 & \text { BDL } & 0.10 \pm 0.03 & \text { BDL } & \text { BDL } & \text { BDL } & 4 \pm 2 & \text { BDL } \\ 0.010-0.018 & \text { BDL } & 0.30 \pm 0.08 & \text { BDL } & \text { BDL } & \text { BDL } & \text { BDL } & \text { BDL }\end{array}$

$\mathrm{BDL}$ = below detection limits

5 Table S4. Mass loadings of amines and ammonium in a fresh biomass burning plume at Burnaby Kensington Park and North Vancouver/Second Narrows sites in British Columbia in July 2015. Relative time is calculated with respect to the local maximum $\mathrm{PM}_{2.5}$ intrusion.

\begin{tabular}{ccccccc}
\hline Sampling & Relative & \multicolumn{5}{c}{ Mass loading $\left(\mathbf{n g ~ m}^{\mathbf{3}}\right.$ ) } \\
\cline { 3 - 7 } site & Time (h) & $\mathbf{N H}_{4}{ }^{+}$ & iMPAH $^{+}$ & TMAH $^{+}$ & DEAH $^{+}$ & TEAH $^{+}$ \\
\hline BKP & -24 & $70 \pm 20$ & $1.4 \pm 0.9$ & $0.6 \pm 0.5$ & $0.9 \pm 0.5$ & $0.2 \pm 0.1$ \\
BKP & -16 & $90 \pm 30$ & $6 \pm 4$ & $3 \pm 2$ & $1.6 \pm 0.8$ & $0.08 \pm 0.06$ \\
BKP & -8 & $130 \pm 40$ & BDL & $5 \pm 4$ & BDL & BDL \\
BKP & 0 & $90 \pm 30$ & $20 \pm 10$ & $9 \pm 7$ & $8 \pm 4$ & BDL \\
BKP & 8 & $90 \pm 30$ & $2 \pm 1$ & $2 \pm 1$ & $1.2 \pm 0.7$ & BDL \\
BKP & 16 & $80 \pm 20$ & $\mathrm{BDL}$ & $\mathrm{BDL}$ & $\mathrm{BDL}$ & $\mathrm{BDL}$ \\
BKP & 24 & $31 \pm 9$ & $\mathrm{BDL}$ & $\mathrm{BDL}$ & $\mathrm{BDL}$ & $\mathrm{BDL}$ \\
NVSN & -24 & $130 \pm 40$ & $5 \pm 3$ & $\mathrm{BDL}$ & $\mathrm{BDL}$ & $\mathrm{BDL}$ \\
NVSN & -16 & $400 \pm 100$ & $14 \pm 9$ & $\mathrm{BDL}$ & $\mathrm{BDL}$ & $\mathrm{BDL}$ \\
NVSN & 0 & $300 \pm 100$ & $40 \pm 30$ & $\mathrm{BDL}$ & $\mathrm{BDL}$ & $\mathrm{BDL}$ \\
NVSN & 8 & $300 \pm 100$ & $60 \pm 40$ & $\mathrm{BDL}$ & $\mathrm{BDL}$ & $\mathrm{BDL}$ \\
NVSN & 16 & $70 \pm 20$ & $\mathrm{BDL}$ & $\mathrm{BDL}$ & $\mathrm{BDL}$ & $\mathrm{BDL}$ \\
NVSN & 24 & $100 \pm 30$ & $\mathrm{BDL}$ & $\mathrm{BDL}$ & $\mathrm{BDL}$ & $\mathrm{BDL}$ \\
\hline
\end{tabular}

